\title{
3 Research Suare \\ Treatment of Aneurysmal Bone Cysts with Titanium Elastic Nails in Children
}

\section{Yi-chen Wang}

Children's Hospital of Shanghai

\section{Xing Jia}

Children's Hospital of Shanghai

\section{Yang Shen}

Children's Hospital of Shanghai

\section{Sun Wang}

Children's Hospital of Shanghai

\section{Liang-chao Dong}

Children's Hospital of Shanghai

\section{Jing Ren}

Children's Hospital of Shanghai

Li-hua Zhao (D 18616771553@163.com )

\section{Research}

Keywords: Primary aneurysmal bone cyst, Titanium Elastic Nails, recurrence, efficacy

Posted Date: July 6th, 2020

DOI: https://doi.org/10.21203/rs.3.rs-38776/v1

License: (c) (1) This work is licensed under a Creative Commons Attribution 4.0 International License.

Read Full License 


\section{Abstract}

Background: The main treatment method of the primary aneurysmal bone cyst (ABC) is to curettage and bone grafts with high-speed burring, radiotherapy, sclerotherapy, arterial embolism and hormone therapy can be used for the lesions whose location cannot be easily exposed by the surgery. Regardless of the method, high recurrence rates are a common problem. The purpose of this study was to evaluate retrospectively the use of titanium elastic nails as a internal fixation in the treatment of aneurysmal bone cysts in children.

Methods: Children with histological primary aneurysmal bone cyst were evaluated between 2010 to 2017 . The patients were divided into 2 groups according to the treatment plan. Patients in the study group operated with curettage and bone grafts with high-speed burring + internal fixation of titanium elastic nails (TEN), and patients in the control group operated with curettage and bone grafts with high-speed burring. The curative effect of the children in the 2 groups were analyzed statistically according to the imaging results (Neer grading) and MSTS functional evaluation.

Results: A total of $26 \mathrm{ABC}$ patients (mean 6.5 years at surgery; 21 males; 5 female) met the inclusion criteria and were evaluated between 2010 to 2017 after a mean follow-up of 5.2 years (range, 2.5-8.4 years), 16 of which in the study group and 10 in the control group. 9 of the 26 patients recurred (3 cases

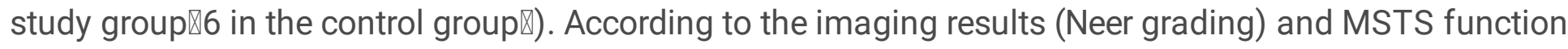
evaluation, there was statistically significant difference in the curative effect between the 2 groups ( $P$ ] 0.05). The recurrence cases in the study group had a better MSTS functioncal recovery.

Conclusion: The use of TEN can significantly improve the cure rate, reduce the recurrence rate and improve the quality of life for children with primary aneurysmal bone cyst.

\section{Background}

Aneurysmal bone cyst (ABC) was original described by Jaffee and Lichtenstein in 1942, which has a low morbidity (about 1.4-3.2/a million people) ${ }^{[1]}$. A large amount of literature and clinical data indicated that $A B C$ is a clinically rare benign bone tumor. However, some researchers presented that $A B C$ is a malignant tumor, an intermediate tumor with malignant tendency ${ }^{[2]}$. The imaging of $A B C$ shows that $A B C$ is a polycystic and expansile osteolytic disorder, $70 \%$ of which are primary, and $30 \%$ are secondary. Up to now, the causes and pathogenic mechanisms of the $A B C$ are still unknown, which leads to the diversified treatment methods of $A B C^{[3-4]}$. Currently, the main treatment method of $A B C$ is curettage and bone grafts with high-speed burring. However, the high recurrence rate of $A B C$ is still a worldwide recognized challenge ${ }^{[5]}$. The recurrence rate of $A B C$ was $11.8-20 \%$, and the interval time between the surgery and the recurrence is 10 months. Some other reports revealed that the recurrence rate was generally higher in young patients than adults, which might be correlated to the location of focus in epiphysis or near epiphysis ${ }^{[6]}$. 
This study retrospectively analyzed the clinical data of children with primary $A B C$ and explored the effectiveness of TEN in the treatment of primary ABC.

\section{Materials And Methods \\ I. Case screening}

The clinical data of the patients who have received surgical treatment after being diagnosed with primary ABC in our Hospital, 2010 to June, 2016 were recorded.

Inclusion criteria: The age of the patients was between 2-14 years old, with foci in the long bones, who have been followed up for more than 2 years, the surgeons who gave them surgical treatment had at least 10 years experience. All the participatences have complete clinical and imaging follow-up data.

Exclusion criteria: patients whose tumor foci were located in the flat bones or short bones, patients who received only plaster immobilization, but not surgical treatment due to pathological bone fracture; patients with secondary ABC.

\section{Treatment Method}

Surgery was performed under general anesthesia.

Control group: curettage and bone grafts with high-speed burring冈plaster immobilization in the upper limbs for 6-8 weeks, and in the lower limbs for 8-12 weeks.

Study group \curettage and bone grafts with high-speed burring and TEN was used under fluoroscopic guidance in an ascending matter (2-C-configuration). The diameter of the TEN (2.0 to $3.0 \mathrm{~mm}$ ) was decided on the basis of the preoperative anterior-posterior radiograph (digitally measured), plaster immobilization in the upper limbs for 4 weeks, and in the lower limbs for 6 weeks.

\section{Follow-up and efficacy evaluation}

All patients were reviewed with clinical examination, X-rays and functional evaluation four to eight weeks after the operation, then every three months until complete bone mineralization occurred and removal of the nails was possible. In order to makesure the long-term effectiveness, we arranged a further evaluation one year after the nails were removed.

Treatment results were classified according to the scheme used by Neer ${ }^{[7]}$ :

- Grade 1 = no response - the cyst showed no evidence of response to the treatment. 
- Grade 2 = recurrence - the cyst initially consolidated with bone, but large areas of osteolysis and cortical thinning subsequently recurred.

- Grade 3 = healed with residual cyst - the cyst was consolidated with bone and the cortical margin thickened but there were still residual cyst parts.

- Grade 4 = healed - the cyst was completely filled in with bone and the cortical margin thickened.

Functional evaluation was performed by MSTS scoring ${ }^{[8]}$. MSTS and radiographic evaluation in the last follow-up time were conducted for both of the treatment groups.

\section{Statistical analysis}

SPSS 20.0 (SPSS company, USA) statistical software package was used for statistical processing. Among them, $\chi 2$ test was used to compare the recurrence rate between the control group and the study group $\varangle \mathrm{R} \times \mathrm{C}$ contingency table $\chi 2$ test was used to compare the prognosis of the two groups, and test of two independent samples was used to compare the MSTS score. Kaplan Meier survival curve was used to analyze the prognosis of the two groups and the relationship between the prognosis and the anatomical position, and the test level a value was 0.05 on both sides.

\section{Results}

\section{General Data}

A total of 26 primary $A B C$ patients were included in the study, and all conformed to the characteristics of typical pathologic histology of $A B C$ (following figure)(Fig.1).

Histological evaluation is mandatory for the accurate diagnosis of $A B C$. Grossly, $A B C$ is spongy, hemorrhagic masses covered by a thin shell of the reactive bone. Microscopically, red blood cells and pale brown hemosiderin are abundant, filling cyst-like spaces bounded by septal proliferations of fibroblasts, mitotically active spindle cells, osteoid, calcifications, and scattered multi-nucleated giant cells ${ }^{[9]}$.

Table 1 showed the specific focus distribution. The content includes 16 cases of the study group and 10 cases of control group; the mean age for the patients of the 2 groups were 5.94 (2-14) years old and 7.20 (2-12) years old, with no statistically significant difference between the 2 groups $(P \otimes 0.05)$.

\section{Prognostic Analysis}

For the patients in the study group, 3 cases for grade I, 0 case for grade II, 1 case for grade III, and 12 cases for grade IV; for the patients in the control group, the prognostic evaluation results of Neer grading 
were 2 cases for grade I, 4 cases for grade II, 3 cases for grade III, and 1 case for grade IV. In addition, there were 2 cases of postoperative pathological fracture in the control group, and 1 case of skin irritation in the study group; none had severe infection, scar formation, or postoperative pathological fracture.

During follow-up period, postoperative MSTS functional scoring was performed for the patients of the 2 groups, and the results showed that the functional score of the patients in TEN treatment group (28.88 \pm $2.22)$ was statistical significantly higher than that of the control group $(19.3 \pm 7.83)(P<0.001)$. According to the postoperative MSTS functional evaluation, we found that patients in the study group were still able to participate physical exercises even though the foci recurring, and no pathological fracture happened, and the postoperative life quality of the patients in the study group was greatly improved (Fig,2) .

\section{Statistical Analysis}

The results of the Chi-square test revealed that there was statistical difference in postoperative NEER grading between the patients of the 2 groups $(P=0.003)$, and the NEER grading prognosis of the patients in the study group was significantly better than that of the control group. According to the literature, the grades I-II of NEER grading were defined as recurrence, and the grades III-IV were defined as cured. Grades III and IV were defined as success, whereas grades I and II represented a failure in treatment. The results of Kaplan-Meier curve and statistical analysis of Log-rank test revealed that there was statistically significant difference between the 2 groups ( $P=0.028$ ) (Fig.3). According to the statistical analysis of the relationship between the recurrence rate and the lesion site in the study group, there was no significant correlation between the lesion site and the recurrence rate $(P=0.092)$ (Fig. 4).

\begin{tabular}{|c|c|c|c|c|c|c|c|c|c|c|c|c|c|c|c|}
\hline \multicolumn{16}{|c|}{ TABLE 1 the condition of two group } \\
\hline Groun & Case number & $A \cos (x)$ & & & & & ion (ca) & & & Distance to the & physis (case/s) & & Rtype at & ry lcast & \\
\hline Group & Case number & Aget(Y) & $M$ & $F$ & radius & humerus & femur & tibia & fibular & metaphysis & diaphysis & 1 & $\|$ & IIII & IV \\
\hline tanium elastic na & 16 & $5.94 \pm 3.30$ & 14 & 2 & 0 & $6 / 37.5$ & $9 / 56.3$ & $1 / 6.2$ & 0 & $13 / 81.3$ & $3 / 188$ & $3 / 1875$ & 0 & $1 / 6.3$ & $12 / 75 \mathrm{f}$ \\
\hline Qurettage & 10 & $7.20+4.87$ & 7 & 3 & $2 / 20.0$ & $2 / 20.0$ & $3 / 30.0$ & 0 & $3 / 30.0$ & $8 / 80.0$ & $2 / 20.0$ & $2 / 20.0$ & $4 / 40.0$ & $3 / 30.0$ & $1 / 10.0$ \\
\hline
\end{tabular}

\section{Discussion}

The standard treatment of $A B C$ is curettage with or without bone-graft. Despite the best efforts at curettage, postoperative highly variable recurrence rates have been shown. As a result, various auxiliary methods have been evolved to reduce the recurrence including the use of cement, high-speed burr, argon beam, phenol and cryotherapy. Most of our patients had disabilities because of the tumor and their treatment, although none of them died or required amputation. It is difficult to treat $A B C$ in the stage of aggressive period. Lesions that occur in the proximal femur should be treated more aggressively, partly because of the high rate of local recurrence and the high risk of fracture. The most appropriate techniques for some of these tumors are curettage surgery and allograft implantation. However, the living quality of the recurred patients significantly reduced ${ }^{[10]}$.

Up to now, the cause of $A B C$ is still unknown. Traditionally, the current consensus believed that it is associated with the pressure increase in local blood vessels. In 1950, Lichtenstein et al ${ }^{[11]}$. proposed that the $A B C$ should not been defined as a bone tumor, but a reactive disease of increased intraosseous 
pressure caused by intraosseous vasogenic disorder (intraosseous phlebemphraxis or arteriovenous fistula). In 1995, Kransdorf et al ${ }^{[12]}$. described the $A B C$ foci as a formation of hemorrhage, and they proposed that continuous bleeding from intraosseous blood capillary created a cavity. Osteolytic change could be a result of rapid expansion of the sclerotin in the lesion area bone cysts formed. According to Mirra et al[ ${ }^{[13]}$.the so-called aneurysmal bone cyst is not a cyst nor a neoplasm; rather, it is probably a periosteal to arteriovenous malformation in bone, not uncommonly seen in association with other wellknown benign and even malignant lesions. In the last 10 years, however, many researchers proposed that the formation of $A B C$ was correlated with gene mutation, and believed that the $A B C$ was a bone tumor, not a disease caused by local bleeding.

Ye $Y$ et al ${ }^{[14]}$. believed that primary $A B C$ has now been identified as an independent neoplasm. The oncogenes responsible for $A B C$ is formed secondary to gain-of-function translocations of $t(16 ; 17)$ (q22;p13) involving a gain-of-function of TRE17/USP6 (ubiquitin-specific-protease USP6 gene). In ABC, this mutation causes the induction of matrix metallopro- teinase (MMP) activity via NF-kB. They think that $A B C$ has no malignant potential although the USP6 gene activatied. Oliveira et al and some Researchers ${ }^{[15-16]}$ believed that primary $A B C$ was a tumor originated from mesenchymal cells, and they found that there was rearrangement of one or two oncogenes in USP6 (ubiquitin-specific protease 6) and $\mathrm{CDH} 11$ (cadherin 11 gene) in the patients with primary $\mathrm{ABC}$, and that there was chromosome translocation in $T(16 ; 17)(q 22 ; p 1)$. They also found that the oncogene USP6 was in a very active state under the regulation of $\mathrm{CDH} 11$ promoter, but there was no translocation of $\mathrm{CDH} 11$ or USP6 in the patients with secondary ABC.

In this study, all of the patients had primary ABC, with good health status evaluated by each system, and without manifestations of malignant tumor. All of the patients of the 2 groups achieved satisfactory efficacies after receiving different treatment protocols, but the efficacy of the study group was superior to that of the control group.

Curettage and bone grafts with high-speed burring is the main treatment of ABC. At present, there were few literatures reported the theoretical basis for the application of TEN in primary ABC. In 2015, Erol B et $\mathrm{al}^{[17]}$. proposed to take the curettage and bone grafts with high-speed burring assisted by the fixation with steel plate, TEN or Kirschner wire, etc. as the treatment method of primary ABC. They found that internal fixation in specific locations can promote healing rate in most of the $A B C$ cases. Classified postoperative NEER grading of the children into 2 classes, namely the recurrence (grade I and II) and cured (grade III and IV).Our data showed that the recurrence rate of the patients in the study group(18.75\%) was significantly lower than that of the control group(60\%), This result indicated that the use of TEN could reduce the recurrence rate $(P<0.05)$. However, the molecular mechanism of which should be further studied in patients with primary ABC. Meanwhile, we also found that the time of postoperative plaster immobilization of the patients in the study group was significantly shorter than that of the control group. Additionally, the patients in the study group did not have pathological bone fracture after recurrence and the result of MSTS functional evaluation of recurrenced cases indicated satisfactory efficacy. According 
to above data, we could find that the internal fixation with TEN can not only increase the cure rate of the patients with primary $A B C$, reduce their recurrence rate, but also significantly reduce the time for postoperative plaster immobilization of the patients and the risk to have another pathological bone fracture, and also significantly improve postoperative life quality of the patients. Meanwhile, we also found that the recurrence factors may be correlated to the lesion location, but statistical analysis indicated no statistical significance. We believe that the use of TEN in the treatment of primary ABC has following advantages: (1) With its good elasticity, each TEN is able to form 3 supporting points in the medullary space; 2 nails are distributed in the medullary space to form double arches, which is a central type internal splint fixation; the mechanical conduction after fixation is a stress sharing mode, which brings less interference to normal biomechanics of the limbs. The nails provide at least 4 kinds of stability of biomechanics, namely counter-bending stability of axial stability, lateral stability and counterrotation stability, which can effectively prevent displacement, angulation and rotation after fixation, therefore, the nails can significantly reduce the time of postoperative plaster immobilization of the patients and improve postoperative life quality of the children. (2) Based on the pathogenesis of the ABC, Biesecker et al ${ }^{[18]}$. supported the hypothesis that $A B C$ was a secondary reactive lesion of bone occurring owing to hemodynamic disturbances based on the results of manometric pressure studies showing increased intracysticpressure. Marcove RC et al ${ }^{[19]}$. suggested that arresting this hemodynamic disturbance could induce healing and prevent recurrence. Healing therefore may occur either spontaneously or after biopsy or fracture. Therefore, we hypothesis that fixation TEN can achieve the effect of continuous intracapsular drainage, and thus reduce intracapsular pressure, promote healing and reduce recurrence. Furthermore, compared with Kirschner wire, TEN is located in the marrow cavity, and it brings less foreign body reaction to surrounding tissues and could reside in body for a long term. (3) After long-term follow-up for the patients of both groups, it was found that all of the recurrenced cases in the study group had no pathological bone fracture, and we believe that when the recurrenced area is located in the stress bone (femur or tibia), the internal fixation with TEN can provide protection and significantly reduce the risk of refracture.

In this study, most of the ABC located at the neck of the femur which is near the metaphysis and is a stress bone in both of the 2 groups. Some researches recommended to curettage and bone grafts with high-speed burring, assisted by internal fixation with steel plate, but local bone cortex of $A B C$ was injured in an osteolytic manner, so it needs to be further discussed whether the internal fixation with steel plate can play a role on the prevention of pathological bone fracture and stabilization once there was a recurrence or expansion of the lesion (Fig. 5). Due to its poor stability, the kirschner wire can hardly play the role of secure internal fixation. Hutchinson $\mathrm{PH}$, Wang $\mathrm{x}$ et $\mathrm{al}^{[20-21]}$ believed that in the treatment of the fracture of the neck of humerus, the penetration of TEN head through the epiphyseal plate of proximal humerus and placement of TEN in the epiphysis would not cause epiphyseal premature closure and affect the growth. For example, we used TEN less than $3 \mathrm{~mm}$ to penetrate the epiphyseal plate of proximal humerus in some cases, and no epiphyseal premature closure was found in the 3 years followup. Due to the mechanism of 4 biological stabilities, the TEN can exert the effect of stability; the cross stress produced by the nails in the medullary space can attain the goal of supporting the longitudinal axis 
of the long bone, and avoid the risk of another pathological bone fracture due to weak sclerotin and lesions following focus recurrence (Fig. 6).

Furthermore, we found one of the patients had the lesion in proximal humerus, which was a active ABC. The patient received curettage and bone grafts with high-speed burring and ESIN. According to our 3 years imaging follow-up. we found that there was a focal recurrence, but the focal recurrence migrated away from the epiphysis as time goes on, and the patient had no obstacles in physical exercise so far, whose score of MSTS was 28 . In 2008, Patrick et al ${ }^{[22]}$. retrospectively analyzed 53 patients with ABC, and found that the patients around 12 years old had a relatively high recurrence rate. 8 out of 19 patients with lesion near the epiphysis had recurrence after the surgery and the recurrence rate significantly higher than the patients with $A B C$ in other locations. This study speculated that this might be correlated with insufficient curettage due to surgeon's concern of postoperative growth deformity during surgery. Therefore, we made a hypothesis that in case of postoperative recurrence, the TEN in the medullary reduced the risk of pathological fracture, and the patient's postoperative functional score and life quality were significantly improved to an extent that he/she could even do physical exercise, and when the patient was older and the focus furtherly migrated away from the epiphysis, another surgical treatment was provided, which, according to our speculation, was able to reduce the recurrence rate and surgical difficulty.

\section{Conclusion}

In summary, according to this study, we believe that the treatment of the primary ABC with curettage and bone grafts with high-speed burring assisted by TEN is a safe and effective treatment measure, which can effectively reduce the recurrence rate, prevent the occurrence of pathological fracture in recurred cases, reduce the time for postoperative plaster immobilization and improve the patient's life quality. However, the number of our cases was limited, further studies are needed based on more and multicenterd cases to prove our conclusion, though statistically significant difference has been got in our study.

\section{Abbreviations}

ABC: Aneurysmal bone cyst

MSTS: musculoskeletal tumor society

TEN: Titanium Elastic Nails

\section{Declarations}

\section{Ethics approval and consent to participate}


The Medical Ethical Commission of the Shanghai Children's Hospital approved this study. Participants signed informed consent form before participation.

\section{Consent for publication}

All authors agree to publish in Medical Science of Monitor

\section{Availability of data and material}

All the data used in the article can be obtained from the medical record information system of Shanghai

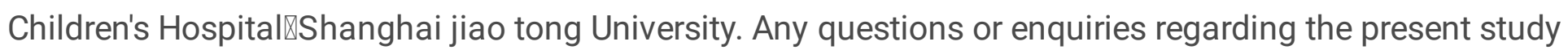
can be directed to Li-hua Zhao, MD (18616771553@163.com), as the corresponding author.

\section{Funding}

The present study was supported by the National Natural Science Foundation of China (no. 81401763), the Health and Family Planning Committee of Shanghai Science Foundation (no. 20144Y0176), the Shanghai Science and Technology Committee (no. 12DZ2295006) and '985 Project' Funds from Shanghai Jiaotong University School of Medicine (no. YBKL2013008).

\section{Acknowledgements}

All authors disclose any financial and personal relationships with other people or organizations that could inappropriately influence their work. There are no conflicts of interest like employment, consultancies, stock ownership, honoraria, paid expert testimony, patent applications/ registrations, and grants or other funding.

\section{Authors' contributions}

Sun Wang,Yang Shen $\varangle$ Liang-chao Dong and Yi-chen Wang were participated in the planning of the study and did the operations. Yi-chen Wang had responsibility for data collection and participated in writing the paper. Li-hua Zhao coordinated the study and had overall responsibility. Yi-chen Wang, Jing Ren , Li-hua Zhao and Xing Jia revised the manuscript critically for important intellectual content. All authors read and approved the final manuscript.

\section{Competing interests}

The authors declare that they have no competing interests. 


\section{Reference}

1. Zehetgruber H, Bittner B, Gruber D, Krepler P, Trieb K, Kotz R, DominkusM.:Prevalence of aneurysmal and solitary bone cysts in young patients.ClinOrthopRelat Res. 2005 Oct;439:136-143.

2. Ahmet Emrah Acan, Onur Basci, Hasan Havitcioglu Aneurysmal bone cyst healing response with intramedullary lengthening nail Acta Orthop Traumatol Turc. 2018 May; 52(3): 232-235. Published online 2017 May 18. doi: 10.1016/j.aott.2017.04.005

3. Percutaneous alcohol-based sclerotherapy in aneurysmal bone cyst in children and adolescents.Orthop Traumatol Surg Res. 2020 Apr 28. pii: S1877-0568(20)30071-2. doi: 10.1016/j.otsr.2019.12.024. Marie-Hardy , El Sayed, Alves , Brunelle , Ouchrif , Naggara , Breton , Mascard, Glorion , Pannier .

4. Hans Roland Dürr , Alexander Klein Aneurysmal bone cyst: results of an off label treatment with Denosumab BMC Musculoskelet Disord. 2019; 20: 456. Published online 2019 Oct 20. doi: 10.1186/s12891-019-2855-y

5. Suat Çelik , Abuzer Uludağ , Hacı Bayram Tosun Unicameral (Simple) and Aneurysmal Bone Cysts: The Effect of Insufficient Curettage on Recurrence PMID: 28154666 PMCID: PMC5267782 DOI: 10.11604/pamj.2016.24.311.9624

6. Keçeci B, Küçük L, Isayev A, Sabah D\&Effect of adjuvant therapies on recurrence in aneurysmal bone cysts. cta Orthop Trauma tol Turc. 2014;48(5):500-506. doi: 10.3944/AOTT.2014.14.0020.

7. Reddy KI, Sinnaeve F, Gaston CL, Grimer RJ, Carter SR. Aneurysmal bone cysts: do simple treatments work? Clin Orthop Relat Res. 2014 Jun;472(6):1901-1910. doi: 10.1007/s11999-014-3513-1. Epub 2014 Feb 15.

8. Enneking WF, Dunham W, Gebhardt MC, Malawar M, Pritchard DJ. A system for the functional evaluation of reconstructive procedures after surgical treatment of tumors of the musculoskeletal system.Clin Orthop Relat Res 1993 Jan;(286):241-246. PMID: 8425352

9. Biesecker JL, Marcove RC, Huvos AG, Miké V. Aneurysmal bone cysts. A clinicopathologic study of 66 cases. Cancer. 1970;26(3): 615-625.

10. Alexandru Ulici, Dan Cosma Aggressive development of an aneurysmal bone cyst of the proximal femur in a paediatric patient: a case report J Int Med Res. 2018 Jan; 46(1): 538-545. Published online 2017 Aug 23. doi: 10.1177/0300060517722244

11. Lichtenstein L: Aneurysmal bone cyst: A pathologic entity commonly mistaken for giant cell tumor and occasionally for hemangioma and osteogenic sarcoma. Cancer 1950;3:279-289.

12. Kransdorf MJ, Sweet DE:Aneurysmal bone cyst: concept, controversy, clinical presentation, and imaging.AJR Am J Roentgenol. 1995 Mar;164(3):573-580

13. Mirra JM (1989) Aneurysmal bone cyst. In: Mirra JM, Picci P, Gold RH (eds) Bone tumors: clinical, radiologic, and pathologic correlations. Lea \&Febiger, Philadelphia, pp 1267-1311

14. Ye Y, Pringle LM, Lau AW, et al. TRE17/USP6 oncogene translocated in aneurysmal bone cyst induces matrix metalloproteinase production via activation of NF-kappaB. Oncogene.2010;29(25):3619- 
3629. doi:10.1038/onc. 2010.116

15. Phillip D. Nicole A. Cipriani Aneurysmal Bone Cyst of the Maxillary Sinus with USP6 Rearrangement: Case Report of a Rare Entity and Review of the Literature Head Neck Pathol. 2019 Sep; 13(3): 281285. Published online 2018 Aug 3. doi: 10.1007/s12105-018-0956-9

16. Oliveira AM, Chou MM. USP6-induced neoplasms: the biologic spectrum of aneurysmal bone cyst and nodular fasciitis. Hum Pathol. 2014 Jan;45(1):1-11. doi:10.1016/j.humpath.2013.03.005. Epub 2013 Jun 12

17. Erol B, Topkar MO, Caliskan E, Erbolukbas R.खSurgical treatment of active or aggressive aneurysmal bone cysts in children. J PediatrOrthop B. 2015 Sep;24(5):461-468. doi:

10.1097/BPB.0000000000000173.

18. Biesecker JL, Marcove RC, Huvos AG, Miké V囚Aneurysmal bone cysts. A clinicopathologic study of 66 cases. Cancer. 1970 Sep;26(3):615-625.

19. Marcove RC, Sheth DS, Takemoto S, Healey JH. The treatment of aneurysmal bone cyst. Clin Orthop Relat Res. 1995 Feb;(311):157-163.

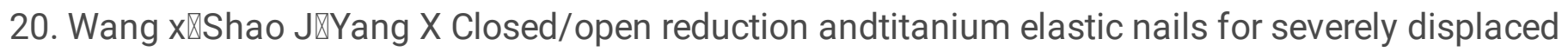
proximal humeralfractures in children[J].IntOrthop $\$ 2014 \llbracket 38(1): 107-110 . D O I: 10.1007 /$ s00264-0132122-z.

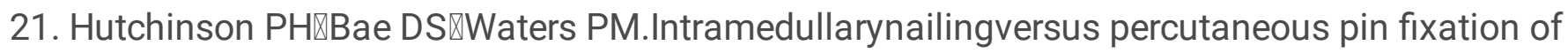
pediatric proximal humerusfractures:a comparison of complications and early radiographic results[J1.J PediatrOrthop $₫ 2011 \llbracket 31(6): 617-622.1301: 10.1097 / B P 0.0 b 013 e 3182210903$.

22. Lin PP, Brown C, Raymond AK, Deavers MT, YaskoAW:Aneurysmal bone cysts recur at juxtaphyseal locations in skeletally immature patients.ClinOrthopRelat Res. 2008 Mar;466(3):722-728. doi: 10.1007/s11999-007-0080-8. Epub 2008 Jan 17.

\section{Figures}

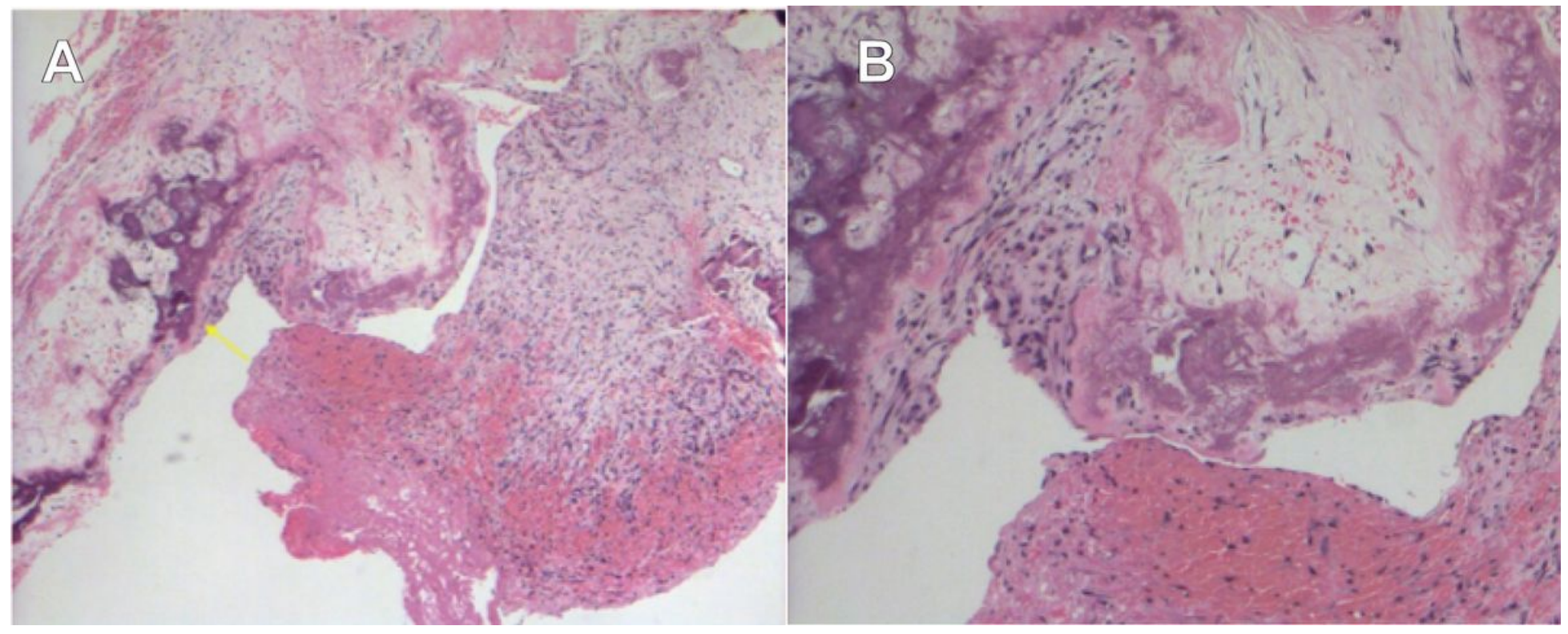




\section{Figure 1}

Histological evaluation is mandatory for the accurate diagnosis of $A B C$. Grossly, $A B C$ are spongy, hemorrhagic masses covered by a thin shell of the reactive bone. Microscopically, red blood cells and often pale brown hemosiderin are abundant, filling cyst-like spaces bounded by septal proliferations of fibroblasts, mitotically active spindle cells, osteoid, calcifications, and scattered multi-nucleated giant cells

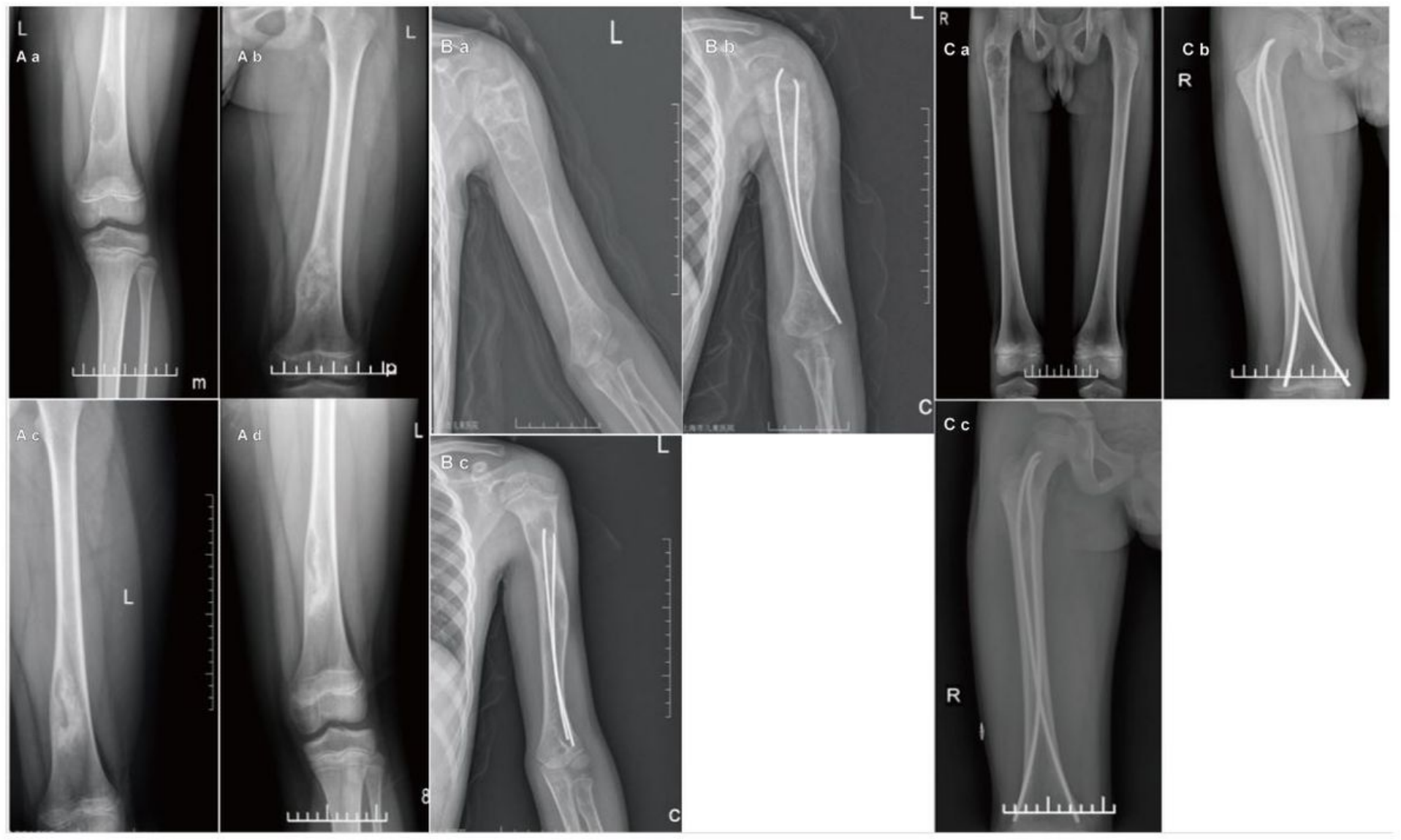

\section{Figure 2}

2A: Neer grade III efficacy evaluation of the group to simply curettage and bone grafts with high-speed burring: postoperative $A B C$ at distal femur reached grade 3 efficacy in Neer grading. 2B: Neer grade III efficacy evaluation of the group to simply curettage and bone grafts with high-speed burring+TEN: postoperative $A B C$ at proximal humerus reached grade 3 efficacy in Neer grading. 2C: Neer grade IV efficacy evaluation of the TEN treatment group: postoperative $A B C$ at the neck of femur (proximal end) reached grade 4 efficacy in Neer grading. 


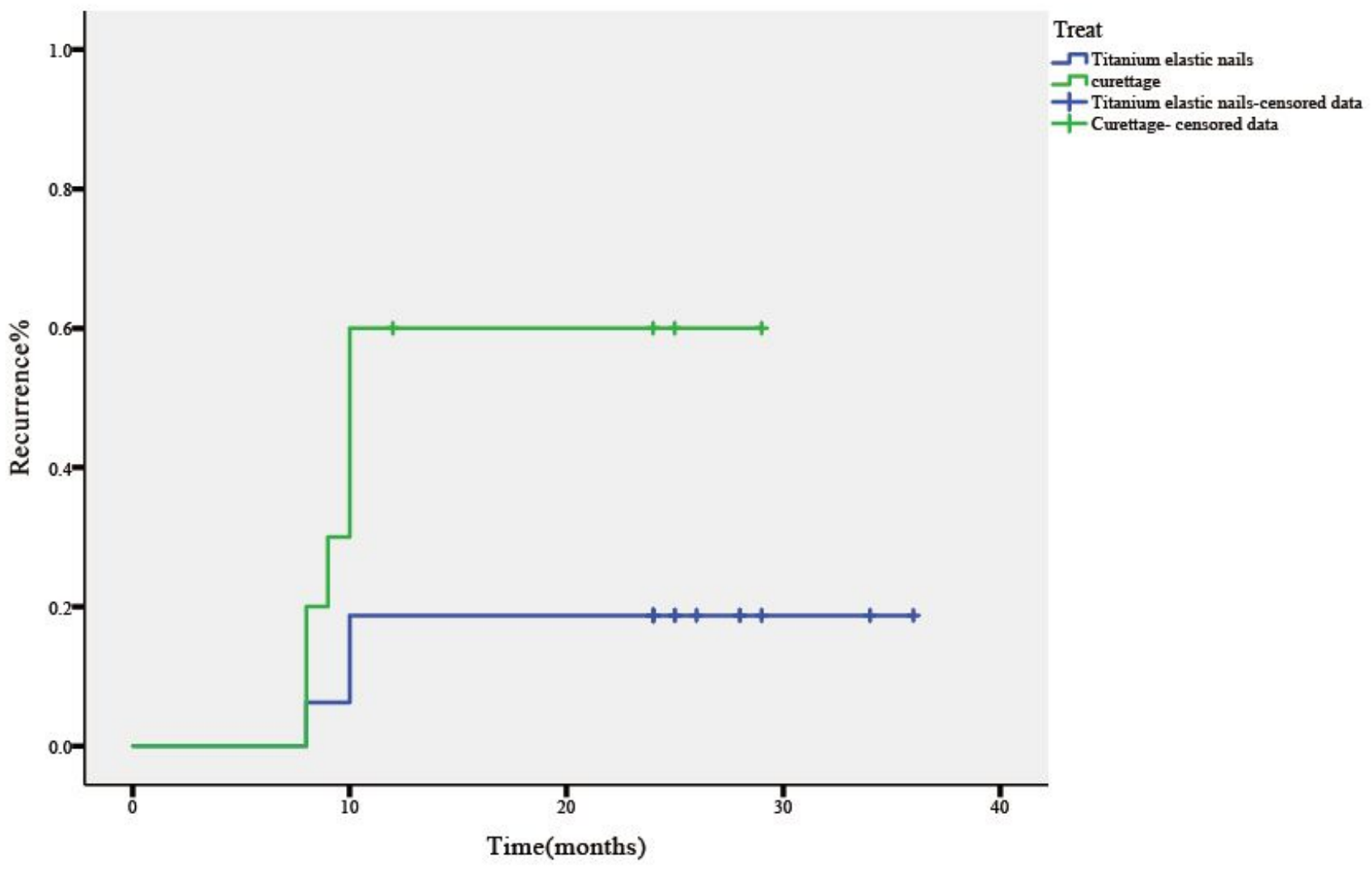

Figure 3

The results of Kaplan-Meier curve and statistical analysis of Log-rank test revealed that there was statistically significant difference between the 2 groups $(P=0.028)$. 


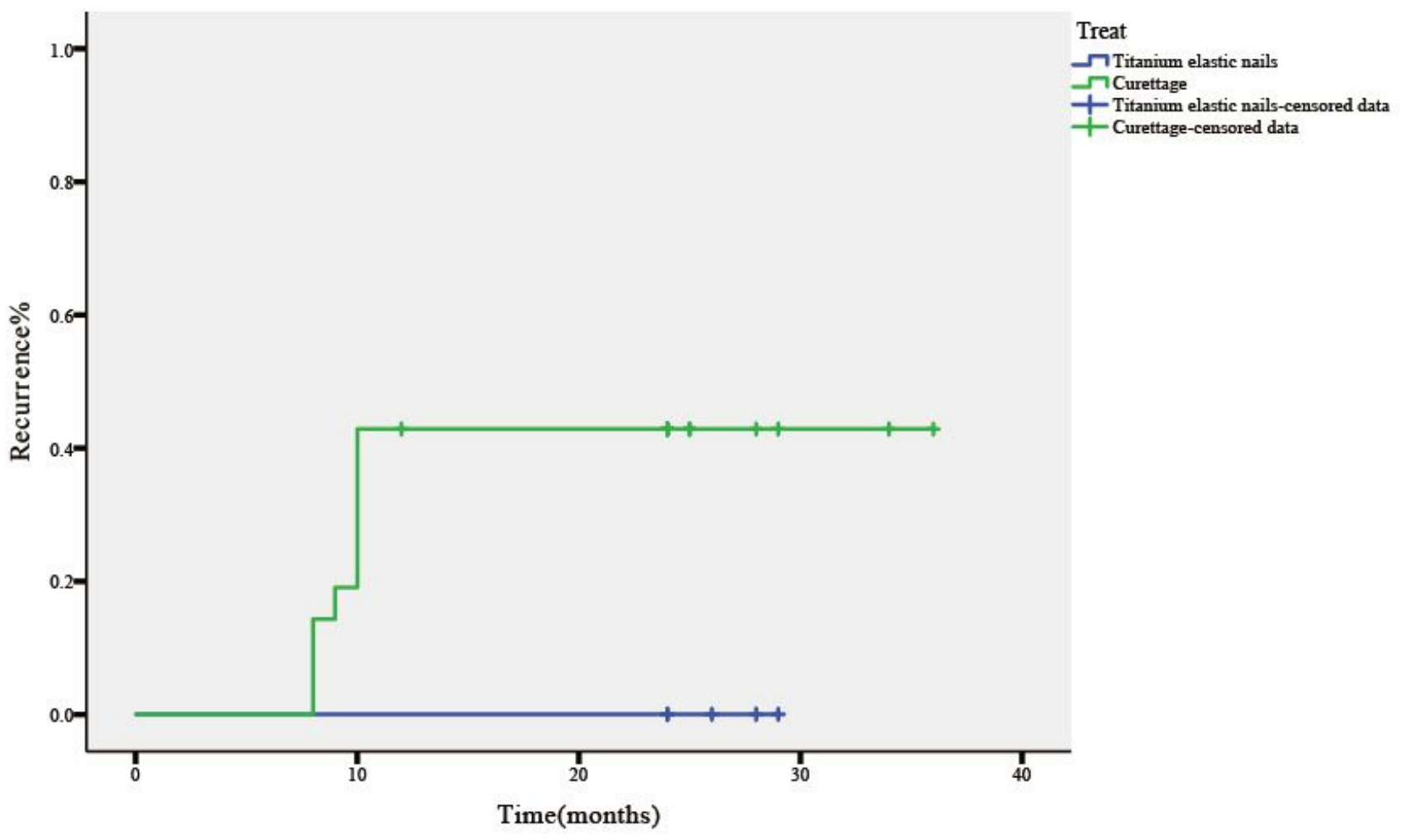

Figure 4

According to the statistical analysis of the relationship between the recurrence rate and the lesion site in the study group, there was no significant correlation between the lesion site and the recurrence rate $(P=$ 0.092) . 


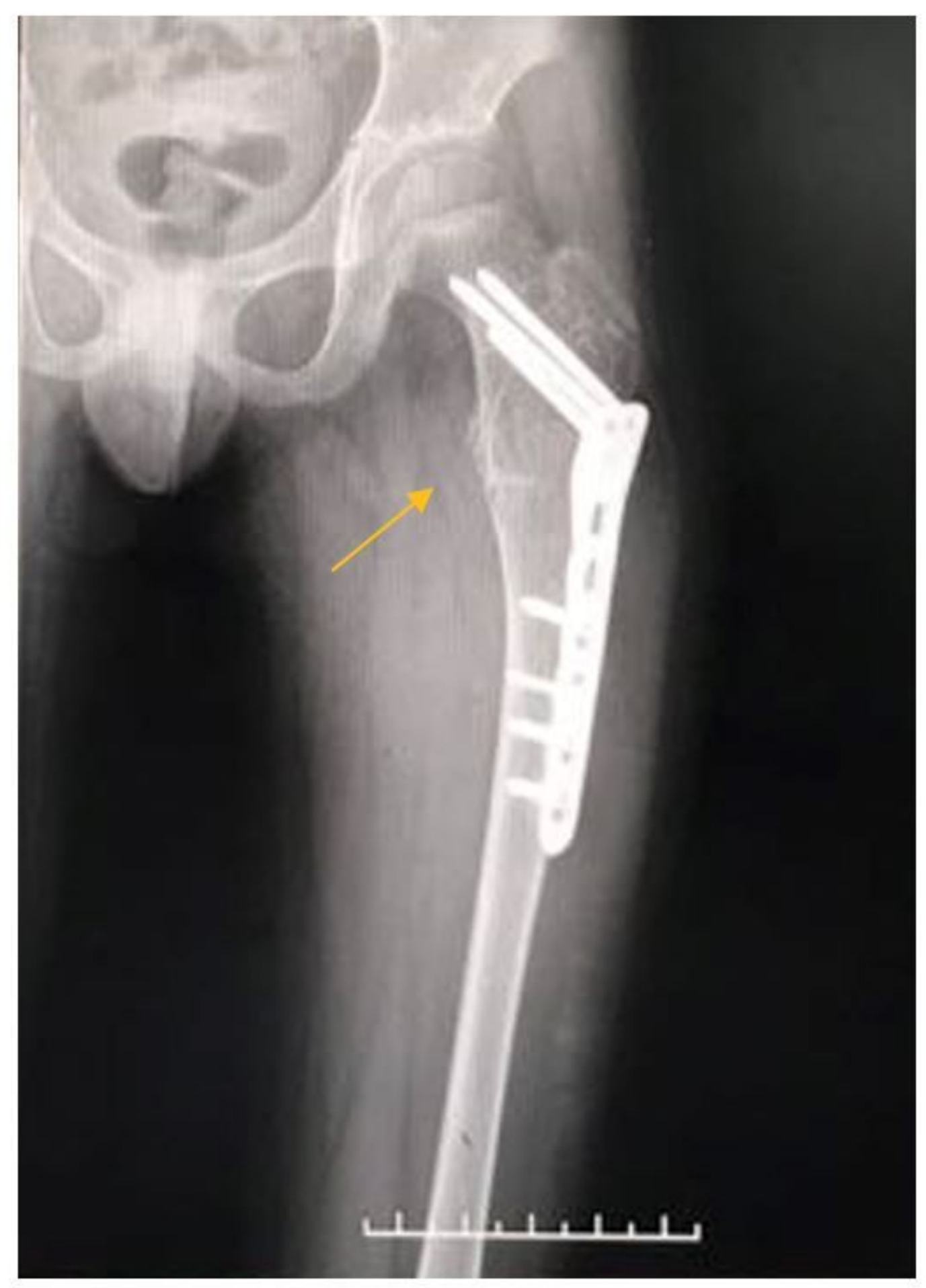

\section{Figure 5}

The patients with recurrence of $A B C$. The figure indicated pathological bone fracture even with internal fixation with steel plate. In view of focal recurrence, osteolytic change of local bone cortex of the neck of femur and cystic change of medullary space, the efficacy of the internal fixation by the 3 nails should be discussed further, and meanwhile unclear fixation efficacy of proximal nail of steel plate led to stress bone fracture. 


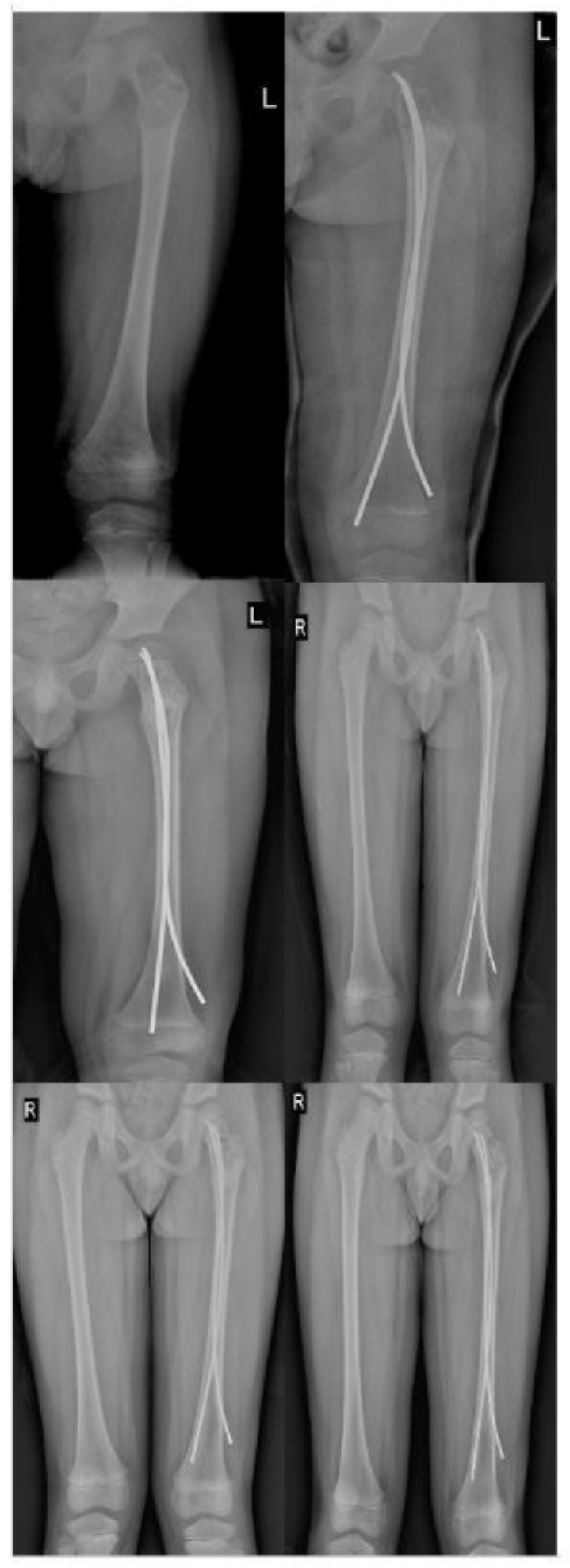

\section{Figure 6}

curettage and bone grafts with high-speed burring + TEN for the foci of neck of femur. The x-rays are present in preoperative, Month 1, 6, 18 and 24 after surgery respectively. The patients of this group had no pathological fracture even after focal recurrence, and no epiphyseal premature closure was observed in 4 years eventhough the penetration of TEN into the epiphyseal plate (4-6 years old), and bilateral lower limbs were equal in length, and the score of MSTS (musculoskeletal tumor society) was 28. 